Talanta 56 (2002) 959-970

Talanta

ELSEVIER

www.elsevier.com/locate/talanta

\title{
Electrochemical detection of in situ adriamycin oxidative damage to DNA
}

\author{
A.M. Oliveira-Brett ${ }^{\mathrm{a}, *}$, M. Vivan ${ }^{\mathrm{b}}$, I.R. Fernandes ${ }^{\mathrm{a}}$, J.A.P. Piedade ${ }^{\mathrm{a}}$ \\ a Departamento de Química, Faculdade de Ciências e Tecnologia, Universidade de Coimbra, 3004-535 Coimbra, Portugal \\ ${ }^{\mathrm{b}}$ Hospital da Universidade de Coimbra, 3000-075 Coimbra, Portugal
}

Received 4 June 2001; received in revised form 3 September 2001; accepted 15 October 2001

\begin{abstract}
Adriamycin intercalation and in situ interaction with double helix DNA was investigated using a voltammetric DNA-biosensor. Oxidation and reduction of adriamycin molecules intercalated in double helix DNA were investigated in order to understand the in vivo mechanism of action with this anti-neoplasic drug. The results showed that the interaction of adriamycin with DNA is potential-dependent causing contact between DNA guanine and adenine bases and the electrode surface such that their oxidation is easily detected. A mechanism for adriamycin reduction and oxidation in situ when intercalated in double helix DNA immobilised onto the glassy carbon electrode surface is presented and the formation of the mutagenic 8-oxoguanine explained. (C) 2002 Elsevier Science B.V. All rights reserved.
\end{abstract}

Keywords: Adriamycin; DNA-biosensor; Oxidative damage; 8-Oxoguanine; Voltammetry

\section{Introduction}

The development of an electrochemical DNAbiosensor that can be used to sense in vivo oxidative damage to DNA is of crucial importance. In a health preventing perspective, detection of in vivo oxidative damage to DNA can be very useful for screening and evaluating the effect caused to DNA by carcinogens [1] and oxidising substances in general, for which voltammetric methods are an inexpensive and faster detection procedure. On the other hand, the interpretation of electrochemical data can contribute to elucidation of the

\footnotetext{
* Corresponding author. Tel./fax: + 351-239-835295.

E-mail address: brett@ci.uc.pt (A.M. Oliveira-Brett).
}

mechanism by which DNA is oxidatively damaged by such substances in an approach to the real action scenario that occurs in the living cell $[2,3]$.

Interactions of several substances with double helix DNA have been successfully studied using a recently-developed voltammetric DNA-biosensor [4,5]. This DNA-biosensor allowed the clarification not only of the specific interaction of some drugs with a certain DNA base but also of the electrochemical mechanism of the interaction [5].

Adriamycin, Scheme 1, is an antibiotic of the family of anthracyclines with a wide spectrum of chemotherapeutic applications and anti-neoplasic action but that causes cardiotoxicity that ranges from a delayed and insidious cardiomyopathy to 


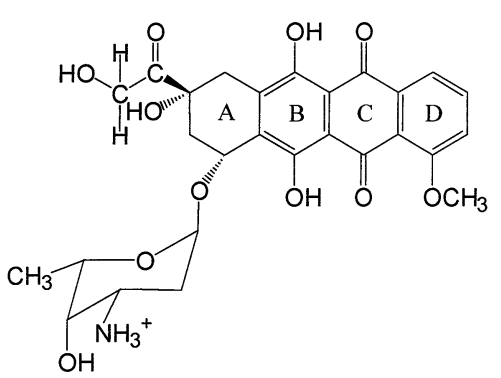

Scheme 1. Adriamycin structure.

irreversible heart failure [1,6-10]. Although, its anti-tumour properties are known for more than 30 years [1,11], its mode of in vivo action is not yet fully understood [10]. Efforts for establishing its mechanisms of action are still an important goal to improve its administration and explain anti-cancer activity.

There is experimental evidence that adriamycin can promote oxidative damage to DNA in cancerous cells through the generation of reactive oxygen species [7-10] and high levels of 8-oxoguanine (8-oxoG), a known biomarker of oxidative stress, were detected in in vitro studies [12]. The generation of this main product of guanine oxidation within DNA is strongly mutagenic and can contribute to cell disfunction [13].

Since 1972 there is ample evidence demonstrated by X-ray crystallography [14] and other methods that adriamycin and analogous anthracycline compounds interact with dsDNA through intercalation [1,15-18] and adriamycin actively accumulates in nuclear genome [19], but less is known as to whether it can directly oxidise dsDNA after intercalation has occurred. In aqueous solution with low salt concentration B-DNA predominates and this is the structure into which intercalation occurs [3].

When intercalated in double helix DNA, the rings $\mathrm{B}$ and $\mathrm{C}$ of the adriamycin core, Scheme 1, intercalate between DNA base pairs preferentially, but not in a sequence specific way, at $\mathrm{CpG}$ homologous sequences [16,17,20,21]. The aminosugar and the carbonyl side chain linked to ring A remain in the minor groove of the double helix and ring D protrudes into the major groove $[14,20,21]$. The positively charged aminosugar in the amino function interacts with the dsDNA phosphate backbone and is fundamental in intercalation and binding affinities with dsDNA [15].

In this context, the aim of the present paper is concerned with the study of the interaction of adriamycin in situ with dsDNA at a charged interface using a dsDNA-modified glassy carbon electrode (GCE). The possibility that adriamycin intercalated to double helix DNA reacts specifically with the guanine moiety and leads to adriamycin-guanine interaction and formation of the mutagenic 8-oxoG is discussed and a mechanism proposed.

The experimental results presented here will give strong evidence that adriamycin can undergo oxidation or reduction after being intercalated into the DNA double helix. Polarographic techniques have been used previously to study the formation of the adriamycin-dsDNA complex in bulk solution [15]. Electrochemical activity for the adriamycin structurally related compound daunomycin intercalated with DNA was also observed using a carbon paste electrode [21]. The results obtained in this work with the dsDNA-modified GCE lead to the conclusion that the electroactive character of the adriamycin-DNA complex depends also on the conformation and orientation of the double helix, parallel or perpendicular, in relation to the electrode surface.

\section{Experimental section}

Adriamycin (Doxorubicin hydrochloride, $2 \mathrm{mg}$ $\mathrm{ml}^{-1}$ solution) obtained from Pharma-APS, and sodium salt calf thymus DNA (type II), 8-oxoG, guanine (G), guanosine (Guo) and adenine (A) from Sigma, were used without further purification. Solutions of different concentrations of adriamycin were prepared in $\mathrm{pH} 4.50 .1 \mathrm{M}$ acetate buffer electrolyte. All solutions were prepared using analytical grade reagents and purified water from a Millipore Milli-Q system (conductivity $<0.1 \mu \mathrm{sm}^{-1}$ ).

Nitrogen and oxygen saturated solutions were obtained by bubbling high purity $\mathrm{N}_{2}$ or $\mathrm{O}_{2}$ in the solution for $10 \mathrm{~min}$ and continuing with a flow of the pure gas over the solution during the voltammetric experiments. 
All voltammetric experiments were done using an $\mu$ Autolab running with GPES version 4.8 software, Eco-Chemie, Utrecht, The Netherlands. A GCE $(d=6 \mathrm{~mm})$ working electrode, a Pt wire counter electrode, and a saturated calomel electrode (SCE) as reference, were used in a $5 \mathrm{~cm}^{3}$ one-compartment electrochemical cell. The experimental conditions unless stated otherwise were: cyclic voltammetry, scan rate $2 \mathrm{~V} \mathrm{~s}^{-1}$; differential pulse voltammetry, pulse amplitude $50 \mathrm{mV}$, pulse width $70 \mathrm{~ms}$, scan rate $5 \mathrm{mV} \mathrm{s}^{-1}$. All potentials are referred to SCE.

Microvolumes were measured using EP-10 and EP-100 Plus Motorized Microliter Pippettes (Rainin Instrument Co. Inc., Woburn, USA). The $\mathrm{pH}$ measurements were carried out with a Crison micropH 2001 pH-meter with an Ingold combined glass electrode. All the experiments were done at room temperature.

ORIGIN (version 6.0) from Microcal Software was used for the presentation of all the experimental voltammograms and graphs reported in this work. When necessary, the experimental voltammograms were smoothed using a SavitskyGolay smoothing algorithm.

\section{Results and discussion}

\subsection{Reduction of adriamycin}

The antibiotic adriamycin, as mentioned earlier, can be oxidised and reduced at a GCE. The reduction of adriamycin occurs at the 5,12diquinone groups in the anthracycline chromophore [22] and the oxidation at the 6,11-dihidroquinone-functionality [23], as shown in the cyclic voltammogram, Fig. 1, where the concentration and scan rate applied are both very high. Both electron transfer reactions are $\mathrm{pH}$ dependent.

The reduction of adriamycin follows a reversible complex mechanism and during reduction a semiquinone radical, which is very reactive with oxygen, is formed. The reduction of the 5,12diquinone group in adriamycin occurs at potentials of -0.40 and $-0.60 \mathrm{~V}$ at $\mathrm{pH} 4.5$, and differential pulse voltammograms show that the

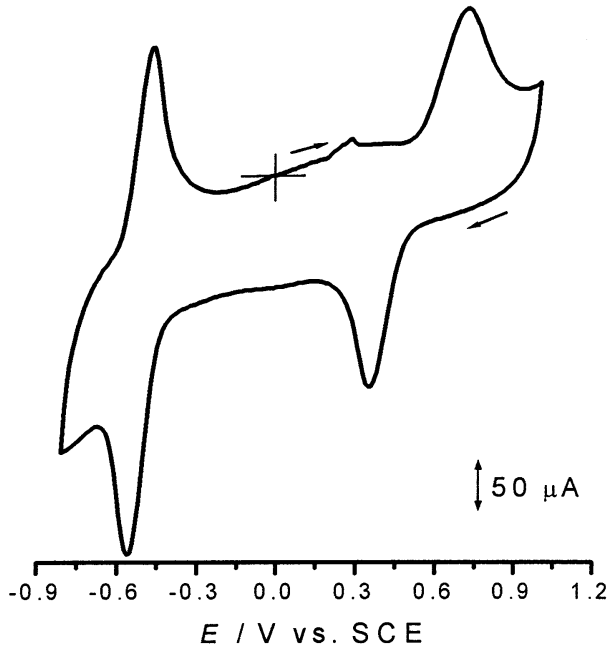

Fig. 1. Cyclic voltammogram of $5 \mu \mathrm{M}$ adriamycin in $\mathrm{pH} 4.5$ $0.1 \mathrm{M}$ acetate buffer obtained with a GCE. Scan rate: $2 \mathrm{~V} \mathrm{~s}^{-1}$. $E_{\mathrm{i}}=0.0 \mathrm{~V}$.

$I_{\mathrm{p}, \mathrm{a}}$ values are dependent on the presence of $\mathrm{O}_{2}$ in solution, Fig. 2. Reversible behaviour is very clear in the absence of oxygen, after nitrogen is bubbled into the solution for $10 \mathrm{~min}$ before scanning.

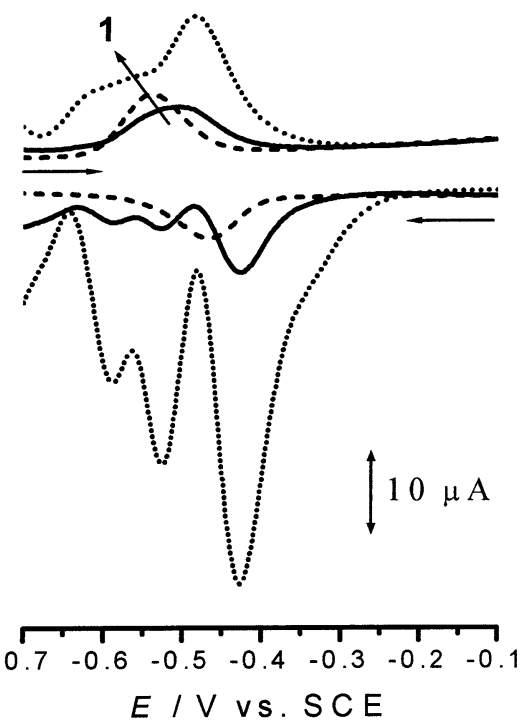

Fig. 2. Background-subtracted differential pulse voltammograms of $1 \mu \mathrm{M}$ adriamycin in $\mathrm{pH} 4.50 .1 \mathrm{M}$ acetate buffer at a bare GCE: normal atmosphere (-); saturated with $\mathrm{O}_{2}$ $(\cdots)$ and $\mathrm{N}_{2}(---)$. Pulse amplitude $50 \mathrm{mV}$, pulse width $70 \mathrm{~ms}$, scan rate $5 \mathrm{mV} \mathrm{s}^{-1}$. 
Multi-step electron transfer occurred in a normal atmosphere or in an oxygen-saturated solution, Fig. 2. In this figure, the reduction and oxidation differential pulse voltammograms were background subtracted and offset so that the reduction and subsequent oxidation processes could be compared. The vertical position of the voltammograms has been altered to make comparison easier.

The catalytic generation of reactive oxygen species (ROS), at $-0.42 \mathrm{~V}$, by adriamycin involves formation of the semiquinone radical intermediate that reduces molecular oxygen to the superoxide radical followed by regeneration of the quinone function moiety, at $-0.47 \mathrm{~V}[15,24]$. This homogeneous adriamycin $-\mathrm{O}_{2}$ redox-cycling process increases ROS generation without adriamycin consumption, occurs in vivo [6-10] and can be detected in situ electrochemically by the significant increase of the reduction, peak at $-0.42 \mathrm{~V}$, and oxidation, peak at $-0.49 \mathrm{~V}$, in the differential pulse voltammograms obtained in saturated oxygen solutions, Fig. 2.

The presence of $\mathrm{O}_{2}$ does not interfere with the oxidation of the 6,11-dihidroquinone functionality [23]. As expected, the oxidation peak current has not changed, Fig. 3a, in the differential pulse voltammograms obtained with the same electrode placed in supporting electrolyte saturated with $\mathrm{N}_{2}$ or with $\mathrm{O}_{2}$. This is important because all the oxidation studies and the electrochemical detection of adriamycin interaction with dsDNA are carried out in contact with normal atmosphere.

\subsection{Adriamycin interaction with DNA}

The electrochemical oxidation at a bare GCE $[4,25]$ of 8 -oxoG, guanine, guanosine, adenine, and dsDNA was briefly revisited in order to make it easier to identify the peaks that occur after adriamycin-dsDNA interaction. The differential pulse voltammograms are shown in Fig. 4. The oxidation peaks for 8-oxoG $\left(E_{\mathrm{p}}=+0.45 \mathrm{~V}\right)$, guanine $\left(E_{\mathrm{p}}=+0.75 \mathrm{~V}\right)$, guanosine $\left(E_{\mathrm{p}}=+0.96 \mathrm{~V}\right)$ and adenine $\left(E_{\mathrm{p}}=+1.05 \mathrm{~V}\right)$, show a good separation between most species. However, for guanosine $\left(E_{\mathrm{p}}=+0.96 \mathrm{~V}\right)$ and adenine $\left(E_{\mathrm{p}}=+\right.$ $1.05 \mathrm{~V})$ there is little difference between the peak
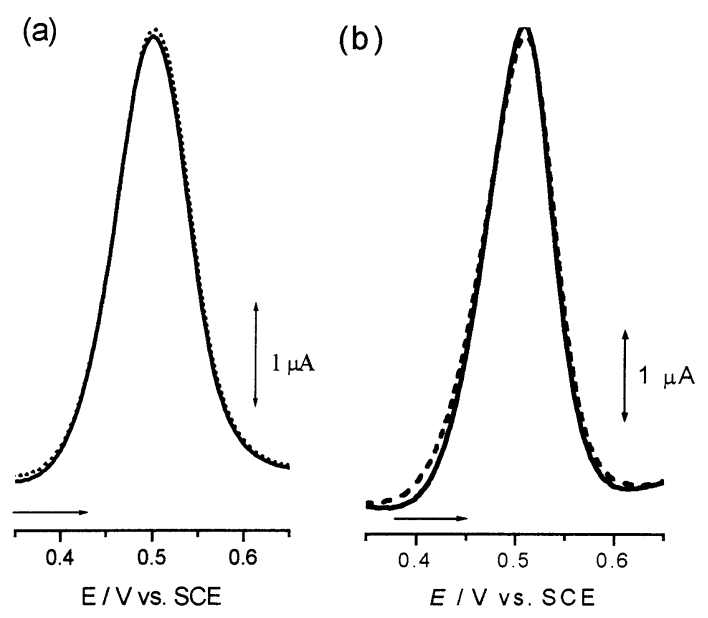

Fig. 3. Differential pulse voltammograms in $\mathrm{pH} 4.50 .1 \mathrm{M}$ acetate buffer at a bare GCE of: (a) $1 \mu \mathrm{M}$ adriamycin saturated with $\mathrm{N}_{2}(-)$ and with $\mathrm{O}_{2}(\cdots)$. First scans; (b) $(-) 1 \mu \mathrm{M}$ adriamycin solution, and (---) adriamycin adsorbed onto the bare GCE and transferred to buffer after rinsing the electrode with deionised water. Pulse amplitude $50 \mathrm{mV}$, pulse width 70 $\mathrm{ms}$, scan rate $5 \mathrm{mV} \mathrm{s}^{-1}$.

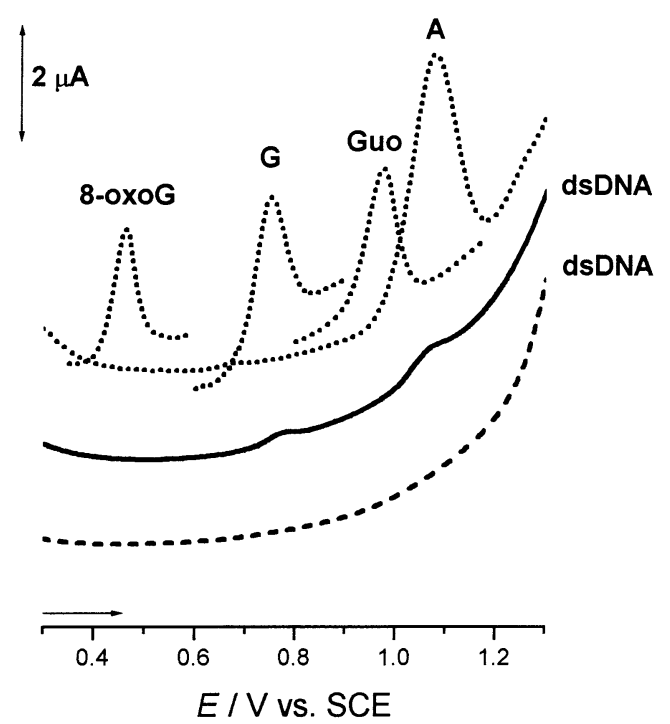

Fig. 4. Differential pulse voltammograms obtained with a bare GCE in pH 4.5 0.1 M acetate buffer of: ( $\cdots) 15 \mu \mathrm{M}$ 8-oxoG;

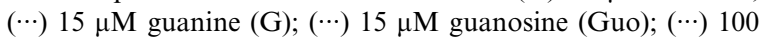
$\mu \mathrm{M}$ adenine (A); and $60 \mu \mathrm{g} \mathrm{ml}^{-1}$ dsDNA (--) first and (-) 40th voltammogram. Pulse amplitude $50 \mathrm{mV}$, pulse width 70 $\mathrm{ms}$, scan rate $5 \mathrm{mV} \mathrm{s}^{-1}$. 
potentials and a considerable peak overlap occurs when both are present in the same solution. This means greater difficulty in their identification when the two species exist together. As already described $[4,5]$ for dsDNA no oxidation peaks were found in the first voltammogram and only after a long adsorption time, the 40th voltammogram, tiny shoulders appeared.

To study electrochemically the adriamycin-dsDNA interaction three different modifications of the GCE were used: a thick layer of dsDNA on the GCE, a thin layer of dsDNA on GCE and adriamycin adsorbed onto GCE.

\subsubsection{Thick layer dsDNA-modified GCE}

A thick layer of dsDNA, $100 \mu$ from a dsDNA concentrated solution $\left(30 \mathrm{mg} \mathrm{ml}^{-1}\right)$, was placed on top of a GCE surface, previously polished and electrochemically conditioned in supporting electrolyte, and allowed to dry [4,5]. This thick layer dsDNA-modified GCE was placed in the solution with adriamycin.

The oxidation of adriamycin at the thick layer dsDNA-modified GCE was investigated, Fig. 5, and the effect of the time of immersion of the modified GCE in $1 \mu \mathrm{M}$ adriamycin solution was compared with the results obtained at a bare GCE. In Fig. $3 \mathrm{~b}$ is shown the voltammogram obtained in buffer for oxidation of $1 \mu \mathrm{M}$ adriamycin accumulated onto the bare GCE by chemisorption [23]. With a bare GCE, Fig. 3a, one adriamycin oxidation peak, $E_{\mathrm{p}}=+0.50 \mathrm{~V}$ vs. SCE, with peak width at half height, $W_{1 / 2}$, close to $91 \mathrm{mV}$, was found by differential pulse voltammetry at $\mathrm{pH} 4.5$. No differences were found for the values of $E_{\mathrm{p}}$ and $W_{1 / 2}$ for a $1 \mu \mathrm{M}$ adriamycin solution Fig. 5a, when using a thick layer dsDNA-modified GCE. However, it was possible to pre-concentrate adriamycin on the thick layer of DNA and the peak current was found to increase with time and to reach saturation after

Fig. 5. Background-subtracted differential pulse voltammograms of $1 \mu \mathrm{M}$ adriamycin in $0.1 \mathrm{M} \mathrm{pH} 4.5$ acetate buffer obtained with a thick layer dsDNA-modified GCE: (a) effect of immersion time, insert $I_{\mathrm{p}}$ vs. $t$; (b) current decrease in successive differential pulse voltammograms: (-) first voltammogram after 5 min immersion, and ( $\cdots)$ fifth voltammogram. Pulse amplitude $50 \mathrm{mV}$, pulse width $70 \mathrm{~ms}$, scan rate $5 \mathrm{mV}$ $\mathrm{s}^{-1}$.
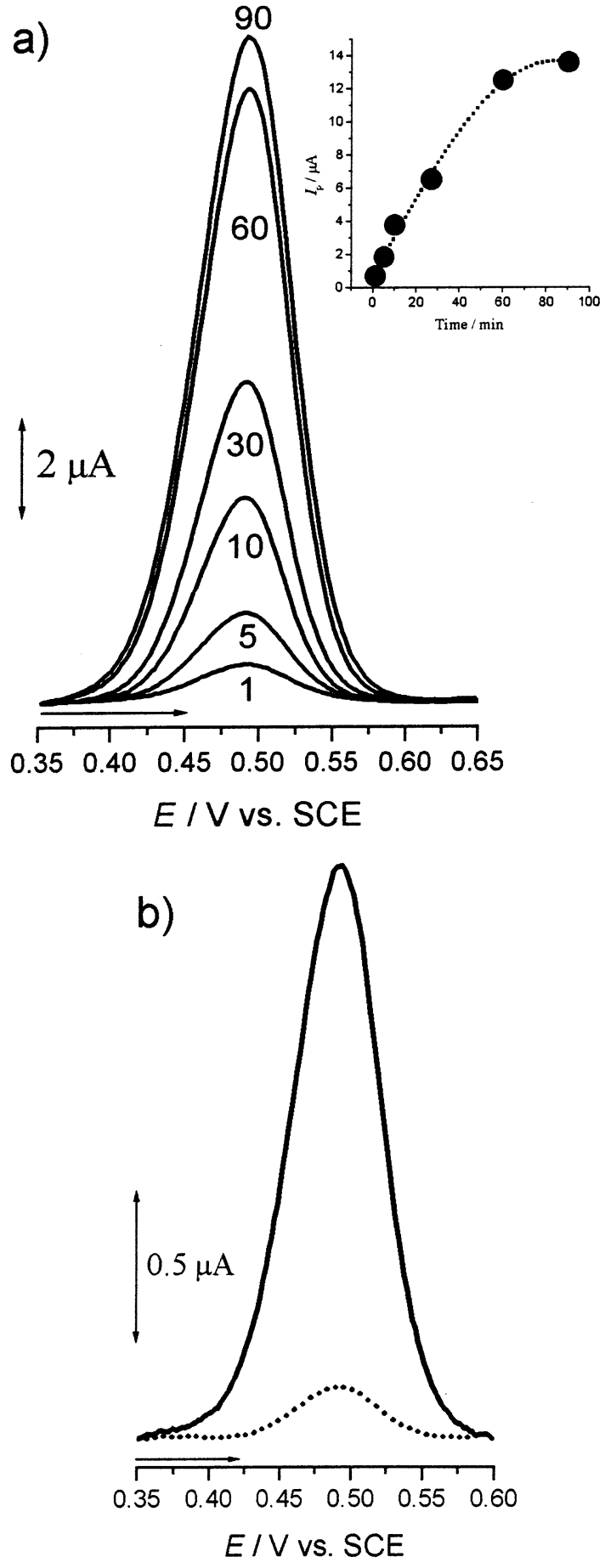
$1 \mathrm{~h}$ of immersion, see insert Fig. 5a and the same occurred with the bare GCE.

Good reproducibility was observed in the values of differential pulse peak current found for the same period of immersion of the thick layer dsDNA-modified GCE in adriamycin solution for the first scan. An abrupt decrease in peak current was always observed in the second scan, Fig. 5b, suggesting a fast consumption of the adriamycin on the surface. This behaviour was found to be reproducible, and a progressive decrease in peak current for the first scan after repeating the $5 \mathrm{~min}$ immersion period was also observed. No identifiable peaks due to DNA base oxidation were registered under these voltammetric conditions.

As discussed, the reduction of adriamycin follows a reversible complex mechanism and during reduction in an oxygen saturated solution the formation of the semiquinone reactive radical resulting from the adriamycin $-\mathrm{O}_{2}$ cyclic catalytic interaction could be followed, Fig. 2.

The thick layer dsDNA-modified GCE was used to study electrochemically the changes in the DNA film caused by the adriamycin radical reacting with $\mathrm{O}_{2}$ formed after in situ reduction of adriamycin. In Fig. 6 are differential pulse voltammograms in acetate buffer electrolyte obtained with a bare GCE immersed in an adriamycin solution and with a thick layer dsDNA-modified GCE previously immersed in an adriamycin solution during $10 \mathrm{~min}$, in a normal atmosphere. In the latter case, before introduction in the acetate buffer solution the modified electrode was gently washed with deionised water to assure the removal of adriamycin that was not intercalated in the dsDNA film. In this way, the voltammetric peaks can only result from adriamycin incorporated into the dsDNA thick film without any contribution from diffusion, as they are obtained in supporting electrolyte. In Fig. 6, the same procedure is used as in Fig. 2, the reduction and oxidation differential pulse voltammograms were background subtracted and offset so that the reduction and subsequent oxidation processes could be compared. The vertical position of the voltammograms has been altered to make comparison easier.

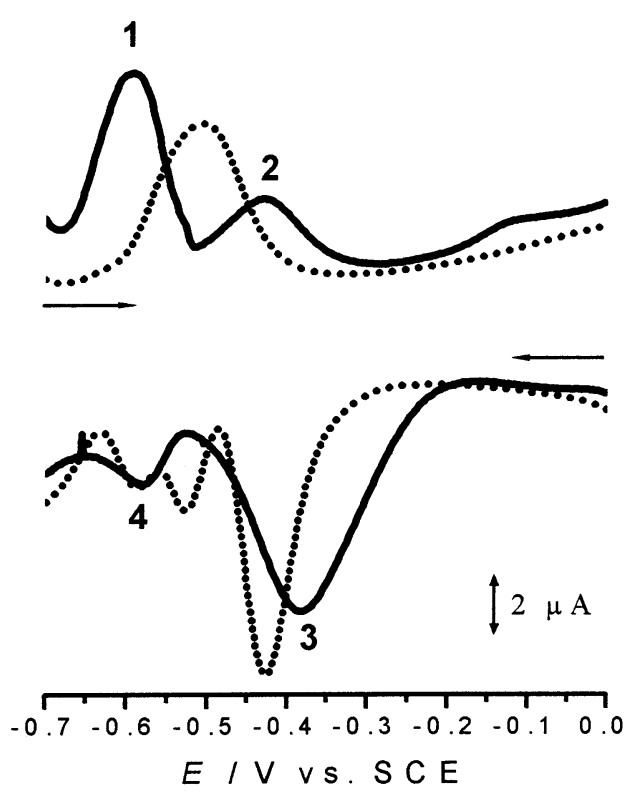

Fig. 6. Background-subtracted differential pulse voltammograms in normal atmosphere in $\mathrm{pH} 4.50 .1 \mathrm{M}$ acetate buffer: $(\cdots)$ bare GCE in a $1 \mu \mathrm{M}$ adriamycin solution; (-) thick layer of dsDNA-modified GCE after being immersed in a $1 \mu \mathrm{M}$ adriamycin solution during $10 \mathrm{~min}$ and rinsed with water before the experiment and transferred to buffer. Pulse amplitude $50 \mathrm{mV}$, pulse width $70 \mathrm{~ms}$, scan rate $5 \mathrm{mV} \mathrm{s}^{-1}$.

Adriamycin adsorbs irreversibly to the GCE surface and it is always necessary to polish and clean the GCE surface very well. When using the thick layer dsDNA-modified GCE it was not necessary to clean the glassy carbon surface after the removal of the dsDNA film. This is a confirmation that all the adriamycin was intercalated inside the thick layer dsDNA film and did not reach the electrode surface. Therefore, the results shown correspond to the signal obtained from adriamycin that was intercalated into the dsDNA thick layer without any contribution from adriamycin in the bulk solution because the experiment was performed only in supporting electrolyte.

The differential pulse voltammograms for the reduction of adriamycin at a bare GCE, Fig. 2, showed three consecutive peaks, at -0.43 , -0.53 and $-0.58 \mathrm{~V}$, whereas at the thick layer dsDNA-modified GCE, Fig. 6, only two peaks occur, at -0.38 (peak 3 ) and $-0.58 \mathrm{~V}$ (peak 4), 
indicating a different mechanism. In the anodic scan, from -0.70 to $0.00 \mathrm{~V}$, at the bare GCE there is one very broad peak for the oxidation of adriamycin, peak 1 at $-0.55 \mathrm{~V}$, whereas at the thick layer DNA-modified GCE, two broad peaks occur, peak 1 at -0.60 , and a new peak at $-0.45 \mathrm{~V}$, peak 2, Fig. 6.

Comparing Fig. 6 with the results presented in Fig. 2, the new peak 2 at $-0.45 \mathrm{~V}$ can be attributed to the adriamycin-guanine site interac-
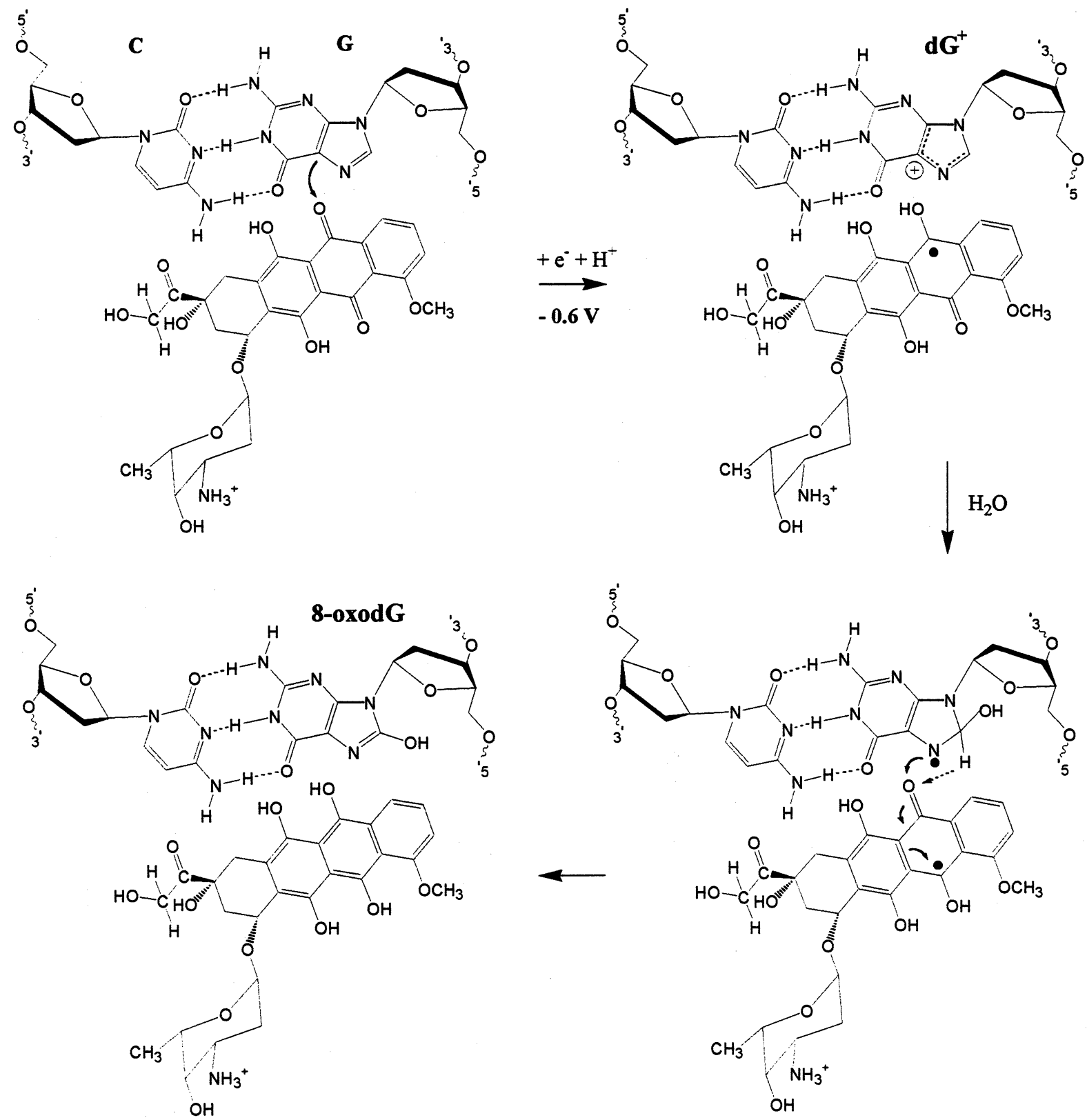

Scheme 2. Mechanism of electrochemical in situ adriamycin oxidative damage to DNA. 


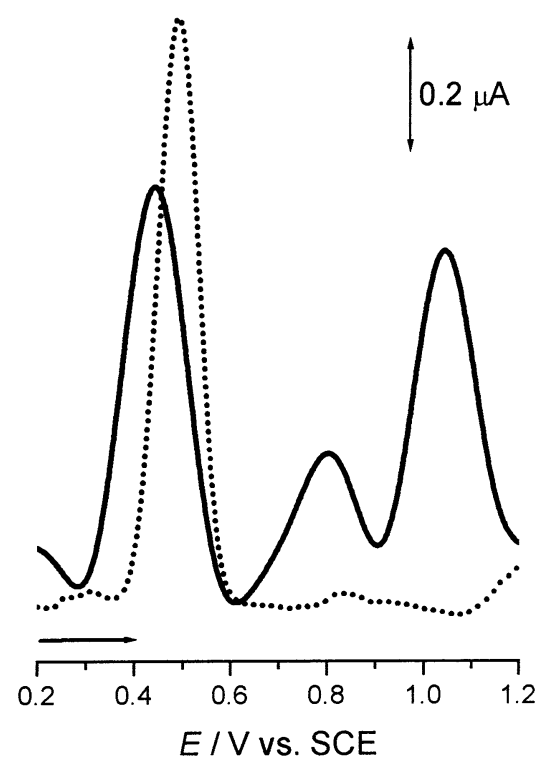

Fig. 7. Background-subtracted differential pulse voltammograms in $\mathrm{pH} 4.50 .1 \mathrm{M}$ acetate buffer obtained with a thick layer dsDNA-modified GCE after being immersed during 10 min in a $1 \mu \mathrm{M}$ adriamycin solution and rinsed with water before the experiment in buffer: $(\cdots)$ without applied potential; (-) subsequent scan after applying a potential of $-0.6 \mathrm{~V}$ during $120 \mathrm{~s}$. Pulse amplitude $50 \mathrm{mV}$, pulse width $70 \mathrm{~ms}$, scan rate $5 \mathrm{mV} \mathrm{s}^{-1}$.

tion in the DNA, resulting in a charge transfer reaction that leads to formation of a guanine radical cation and adriamycin semiquinone, Scheme 2. In Fig. 6, using the thick layer dsDNAmodified GCE, peak 1 occurs at $-0.60 \mathrm{~V}$, and corresponds to the oxidation of the free adriamycin reduced quinone group, peak 1 at -0.55 $\mathrm{V}$ in Fig. 2, that is shifted to more negative potential values due to the electrode modification with dsDNA.

As mentioned the oxidation peak potential of the 6,11-dihidroquinone-functionality showed no difference when a thick layer dsDNA-modified GCE was used, Fig. 5, and it was even possible to concentrate adriamycin onto the DNA thick layer although no interaction with DNA was observed, independently of the time of immersion.

However, if a potential of $-0.60 \mathrm{~V}$ was applied to the dsDNA-modified electrode during $120 \mathrm{~s}$, big changes occurred inside the DNA layer, Fig. 7. Two new oxidation peaks appeared, that can be identified [4]: the first at $+0.80 \mathrm{~V}$, as guanine oxidation, and the second at $+1.05 \mathrm{~V}$, most probably as adenine oxidation, Fig. 4. Nevertheless, the oxidation peak potentials for guanosine and adenine are very close, Fig. 4, and this makes their identification difficult. As adriamycin intercalates between DNA base pairs preferentially at $\mathrm{CpG}$ homologous sequences $[16,17,20,21]$ is understandable that peaks for guanine and guanosine will occur. However, it is also possible that adenine molecules in the unfolded strand reach the electrode. The broad peak observed probably includes a contribution from both guanosine and adenine, but it is not possible to obtain two separate peaks.

Adriamycin can undergo a reaction beginning with the transfer of a single electron to the quinone portion of the adriamycin ring system, generating a free radical [1]. A mechanism of guanine oxidation, after adriamycin intercalation within double helix DNA [14,16], by the very reactive adriamycin radical formed at $-0.60 \mathrm{~V}$ is proposed in Scheme 2 and is in agreement with results obtained by other methods $[1,7,12,18]$. Besides these two new peaks, the oxidation peak of adriamycin became broader, the peak width at half height, $W_{1 / 2}$, being greater than that obtained for adriamycin, and appeared at $E_{\mathrm{p}}=+0.45 \mathrm{~V}$, a peak potential $50 \mathrm{mV}$ less positive than for adriamycin alone, shifted towards the potential for 8-oxoG oxidation, Fig. 4 [25]. Following Scheme 2 , this peak can be related with the interaction between adriamycin and 8-oxoG formed after adriamycin intercalation in DNA in guanine-rich regions $[16,20]$.

\subsubsection{Thin layer dsDNA-modified GCE}

A thin layer dsDNA-modified GCE was prepared by immersing the GCE in a $60 \mu \mathrm{g} \mathrm{ml}-1$ dsDNA solution at $+0.40 \mathrm{~V}$ applied potential during $10 \mathrm{~min}$, a similar procedure as has been used with ssDNA on carbon paste electrodes [26]. In this electrode modification a non-uniform thin layer of dsDNA is adsorbed onto the GCE leaving many bare glassy carbon uncovered regions. This thin layer dsDNA-modified GCE was immersed during $3 \mathrm{~min}$ in an adriamycin solution, rinsed with water, and afterwards transferred to 
buffer, where differential pulse voltammetry was performed. Results in acetate buffer, Fig. 8, showed different features. Without applying a negative potential only the peak for adriamycin oxidation at $+0.50 \mathrm{~V}$ occurs. After applying the potential of $-0.60 \mathrm{~V}$ during $60 \mathrm{~s}$, the oxidation peak for guanine, at $+0.84 \mathrm{~V}$, and the oxidation peak for 8-oxoG in the unfolded strand, at $+0.38 \mathrm{~V}$, also appeared.

These results are in agreement with those with the thick layer dsDNA-modified GCE. The clear separation of the adriamycin and 8-oxoG peaks can be explained by the non-uniform coverage of the electrode surface by DNA and adsorption of adriamycin on the uncovered glassy carbon. This is very important as it enables the use of the less positive peak of oxidation of 8 -oxoG to detect DNA damage, which can only be achieved with the thin layer dsDNA-modified GCE.

However, it is not possible to detect the adriamycin-DNA damage by monitoring only changes in the adriamycin oxidation peak. The

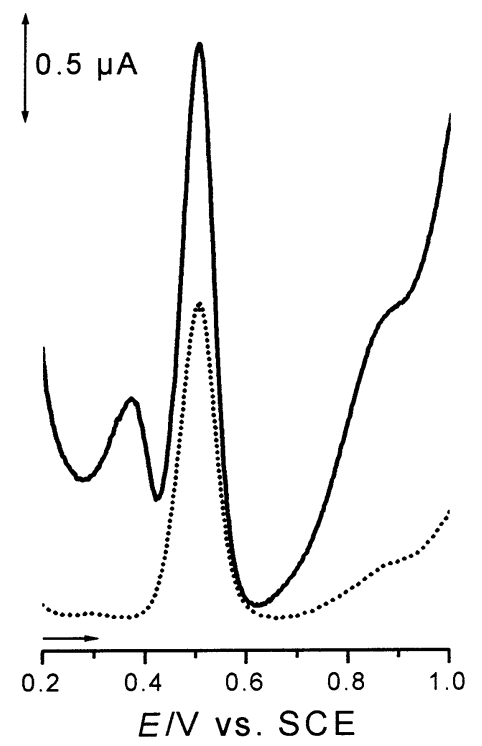

Fig. 8. Differential pulse voltammograms in $\mathrm{pH} 4.50 .1 \mathrm{M}$ acetate buffer obtained with a thin layer dsDNA-modified GCE after being immersed in a $5 \mu \mathrm{M}$ adriamycin solution during $3 \mathrm{~min}$ and rinsed with water before the experiment in buffer: ( $\cdots$ ) without applied potential; (-) after applying a potential of $-0.6 \mathrm{~V}$ during $60 \mathrm{~s}$. Pulse amplitude $50 \mathrm{mV}$, pulse width $70 \mathrm{~ms}$, scan rate $5 \mathrm{mV} \mathrm{s}^{-1}$. First scans. damage to immobilised dsDNA causes the appearance of oxidation peaks from DNA bases and this should always be measured and taken into account. In fact, this is the drawback of the thin layer dsDNA-modified GCE since it leads to two contributions from simple adsorbed analyte and damage to immobilised DNA which needs to be carefully distinguished.

\subsubsection{Adriamycin adsorbed-modified GCE}

It is interesting to take advantage of adriamycin's strong and irreversible adsorption on the glassy carbon surface. In Fig. 3b, the voltammogram in buffer shows the accumulation of adriamycin onto a bare GCE. An adriamycin adsorbed-modified GCE could be prepared by immersing the electrode in an adriamycin solution for a short period of time with or without deposition potential applied. To prepare the adriamycin adsorbed-modified GCE used here the GCE was immersed in a $5 \mu \mathrm{M}$ adriamycin solution for $10 \mathrm{~min}$ at a deposition potential of $+0.40 \mathrm{~V}$, and was then always rinsed thoroughly with water before transferring to the DNA solution where the voltammetric measurements were done. As before, the reduction of the chemisorbed adriamycin [23] was always investigated and the voltammograms were obtained in buffer solution without adriamycin in solution so as to have no contribution from bulk analyte diffusion. The effect of the presence of $\mathrm{O}_{2}$ (supporting electrolyte saturated with $\mathrm{O}_{2}$ ) and absence (supporting electrolyte saturated with $\mathrm{N}_{2}$ ) on the voltammetric behaviour of the adsorbed layer of adriamycin was also investigated. The results confirmed the strong dependence of the reduction reaction of adsorbed adriamycin on the presence of oxygen, and no influence on the oxidation, described earlier with adriamycin in solution, Figs. 2 and 3a, respectively.

At the adriamycin adsorbed-modified GCE, adriamycin was confined to the electrode surface and the total surface concentration of adriamycin was calculated using,

$\Gamma_{\mathrm{adr}}=4 R T I_{\mathrm{p}, \mathrm{a}} / n^{2} F^{2} A v$,

to be $\Gamma_{\mathrm{adr}}=2.57 \times 10^{-10} \mathrm{~mol} \mathrm{~cm}^{-2}$ [27]. 


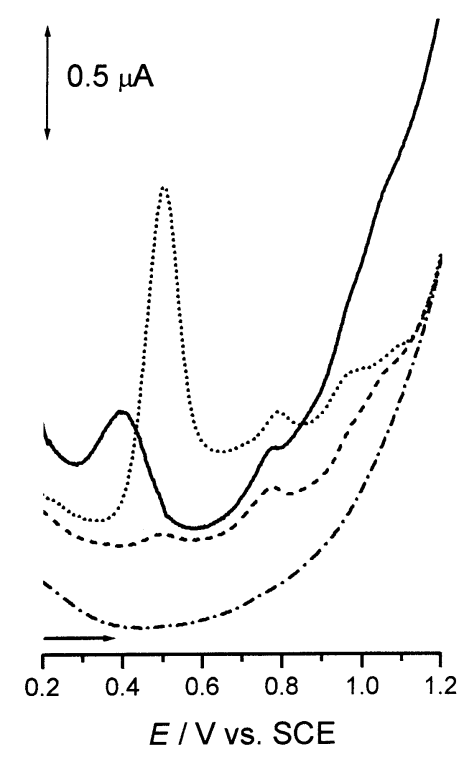

Fig. 9. Background-subtracted differential pulse voltammograms in a dsDNA $60 \mu \mathrm{g} \mathrm{ml}^{-1}$ solution in $\mathrm{pH} 4.50 .1 \mathrm{M}$ acetate buffer obtained with: a bare GCE (...-) after applying a potential of $-0.6 \mathrm{~V}$ during $60 \mathrm{~s}$; and an adriamycin adsorbed-modified GCE: ( $\cdots)$ first scan with no potential applied; (--) after applying a potential of $0.0 \mathrm{~V}$ during $60 \mathrm{~s}$; $(-)$ after applying a potential of $-0.6 \mathrm{~V}$ during $60 \mathrm{~s}$. Pulse amplitude $50 \mathrm{mV}$, pulse width $70 \mathrm{~ms}$, scan rate $5 \mathrm{mV} \mathrm{s}^{-1}$.

The interaction with DNA in solution was investigated by differential pulse voltammetry, Fig. 9. The first scan shows a big peak corresponding to the oxidation of the adsorbed adriamycin and other peaks attributed to DNA purine bases oxidation can also be seen. This is indicative that even adriamycin chemisorbed to the electrode surface intercalates with DNA that diffuses from solution, causing distortion of the double strand and allowing the oxidation of the purine bases.

A subsequent differential pulse voltammogram obtained after application of $0.00 \mathrm{~V}$ for $60 \mathrm{~s}$ shows a drastic decrease in the adriamycin oxidation peak, Fig. 9, besides the peaks already mentioned for the oxidation of the purine bases. This decrease in the adriamycin oxidation peak is explained easily because the only adriamycin existing on the electrode surface has reacted intercalating with DNA, so the 6,11-dihydroquinone functionality is not available any more to react at the electrode surface.
However, if after the first scan a potential of $-0.60 \mathrm{~V}$ is applied during $60 \mathrm{~s}$, a broad peak, at $+0.40 \mathrm{~V}$, appears, Fig. 9. This peak can be identified, as in Fig. 7, with the interaction between adriamycin and 8-oxoG formed after adriamycin intercalation in DNA in guanine-rich regions $[16,20]$, in agreement with the proposed mechanism, Scheme 2.

\subsection{Adriamycin in situ oxidative damage to DNA}

The adriamycin reduction clearly conditions the adriamycin-dsDNA interaction and this was shown using the three different modifications of the GCE: a thick layer of dsDNA onto GCE, a thin layer of dsDNA onto GCE and adriamycin adsorbed onto GCE.

Adriamycin reduction occurs inside the dsDNA thick layer and the radical formed can interact with DNA in situ and the products be retained in the DNA layer. The reaction of $\mathrm{O}_{2}$ with adriamycin at the electrode surface should also be considered. It is not expected that the concentration of $\mathrm{O}_{2}$ in the thick layer dsDNA-modified GCE is sufficiently large to explain the similar peak split observed in $\mathrm{O}_{2}$-saturated solutions. Therefore, this must be explained by considering that the reduced adriamycin reactive radical interacts with nucleotides of the DNA in which it is intercalated in a similar fashion to the adriamycin- $\mathrm{O}_{2}$ mechanism which occurs in $\mathrm{O}_{2}$-saturated solutions. Thus, this reduced adriamycin radical interaction with DNA is responsible for the changes in DNA, always detected after the conditioning potential of $-0.60 \mathrm{~V}$ was applied. In fact, the thick-layer dsDNA-modified GCE is a multi-layer film of DNA gel covering the glassy carbon surface completely and uniformly with no pinholes or GCE uncovered regions. Since the experiments were carried out in buffer, the peaks recorded for the reduction or oxidation of adriamycin can only be attributed to the reaction of adriamycin molecules that are inside the thick film of dsDNA.

The results presented here give experimental electrochemical evidence that adriamycin intercalates in double helix DNA and can oxidatively damage DNA, leading to the generation of 8- 
oxoG. Electrochemical in situ generation of adriamycin semiquinone radical at $-0.60 \mathrm{~V}$ permits a detailed study of this interaction mechanism. In Scheme 2 is shown the proposed mechanism for the generation of 8-oxoG when a negative conditioning potential is applied, by intercalation of adriamycin into the double helix DNA.

In the configuration of Scheme 2 both adriamycin electroactive functional groups (the oxidisable hydroquinone group in ring $\mathrm{B}$, and the reducible quinone function in ring $\mathrm{C}$ ) are intercalated between the base pairs in the DNA-adriamycin complex in close interaction with the GC base pair. The reducible quinone group in ring $\mathrm{C}$ protrudes slightly into the major groove, and this enables in situ (in helix) generation of an adriamycin radical within the double helix. Therefore, a redox reaction between adriamycin and guanine residues inside the double helix of DNA can be considered, in order to explain the experimental data.

8-Oxoguanine is the main product of guanine oxidation, but the mechanistic pathway involving two electrons and two protons [25] depends on the chemical environment surrounding guanine. Contrary to the oxidation of free guanine in solution, in the biopolymer, deprotonation of the guanine radical cation is partially prevented by base-stacking interactions with cytosine, and the hydrolysis reaction is most favourable to occur in double helix DNA [28].

The formation of adriamycin-DNA adducts has been reported $[29,30]$ and the hypothesis that their formation involves redox reactions between intercalated adriamycin and base pairs must be taken into account in the light of the present results. In fact, the transitory generation of the guanine radical cation close to the adriamycin semiquinone radical cation may result in an adriamycin-base adduct formation that competes with the hydrolysis step. The intercalation may cause sufficient distortion of the dsDNA to expose the radical cation to hydrolysis. This would lead to the transient formation of a 8-hydroxy-7,8-dihydropurin-7-yl radical, the likely precursor of 8oxoG inside the double helix [31,32].

When a potential of $-0.6 \mathrm{~V}$ is applied, adriamycin is reduced and this redox process occurs within the DNA double helix and involves the simultaneous oxidation of one neighbouring guanine residue. In this way, electron transfer from the guanine moiety to the quinone without hydrogen abstraction is likely to be the predominant reaction leading to the formation of the guanine cation. Due to the fast hydrolysis of the radical cation, the semiquinone undergoes further reduction to the fully reduced adriamycin and the formation of 8-oxoG occurs, instead of being reoxidised, as would happen in $\mathrm{O}_{2}$-saturated solution. So the hydrolysis step is followed by a second electron transfer and leads to the ultimate formation of 8-oxoG and reduced adriamycin.

Finally, the $\pi$-stacked base pairs characteristic of double helix DNA might serve as a pathway for charge transport $[33,34]$ mediating the redox reaction between adriamycin radicals, generated during reduction, and guanine residues in the double helix. The trapping by water of the generated radical cation leads to in situ formation of the oxidative DNA damage product 8-oxoG. Further oxidation of 8-oxoG could lead to hydrolysis of the glycosidic bond generating an abasic site [28]. This could disrupt the double helix causing strand break, exposing 8-oxoG residues and other purines to the electrode surface that can then be oxidised.

\section{Conclusions}

Electrochemical voltammetric in situ sensing of DNA oxidative damage caused by reduced adriamycin intercalated into DNA was possible using the dsDNA film-modified GCE. The results indicate that adriamycin intercalated in double helix DNA can undergo oxidation or reduction and reacts specifically with the guanine moiety, and thence to the formation of mutagenic 8-oxoG residues. A model for this electrochemically observed in situ oxidative damage was proposed and may be used to explain the levels of 8-oxoG found when cells are treated with adriamycin as well as its well-known free radical activity.

This is very relevant because the mechanism of interaction of DNA-adriamycin at charged interfaces mimics better the in vivo DNA-adriamycin 
complex situation, where it is expected that DNA is in close contact with charged phospholipid membranes and proteins, rather than when the interaction is in solution. The results presented contribute with a possible mechanism through which adriamycin can cause direct in vivo oxidative damage to DNA after intercalation.

The potential use of this DNA film-modified GCE for the understanding of DNA interactions with molecules or ions explores, in a promising way, the use of voltammetric techniques for in situ generation of reactive intermediates and is a complementary tool for the study of biomolecular interaction mechanisms.

\section{Acknowledgements}

Financial support from Fundação para a Ciência e Tecnologia (FCT) projects PRAXIS/C/SAU/ 93/96 and POCTI/33305/99 (co-financed by the European Community fund FEDER), ICEMS (Research Unit 103) and European Projects ERBICT15-CT960804 and QLK3-2000-01311, are gratefully acknowledged.

\section{References}

[1] M.C. Perry, The Chemotherapy Source Book, Williams and Wilkins, Baltimore, USA, 1996.

[2] G.M. Blackburn, M.J. Gair, Nucleic Acids in Chemistry and Biology, Oxford University Press, UK, 1996.

[3] W. Saenger, Principles of Nucleic Acid Structure, Springer-Verlag, New York, 1984.

[4] A.M. Oliveira-Brett, S.H.P. Serrano, J.A.P. Piedade, in: R.G. Compton, E.G. Hancock (Eds.), Comprehensive Chemical Kinetics, vol. 37, Elsevier, Amsterdam, 1999, pp. 91-119 Chapter 3.

[5] A.M. Oliveira Brett, S.H.P. Serrano, T.A. Macedo, D. Raimundo, M.H. Marques, M.A. La-Scalea, Electroanalysis 7 (1996) 992-995.

[6] E.L. de Beer, A.E. Bottone, E.E. Voest, Eur. J. Pharmacol. 415 (1) (2001) 1-11.

[7] S.Y. Zhou, A. Starkov, M.K. Froberg, R.L. Leino, K.B. Wallace, Cancer Res. 61 (2) (2001) 771-777.

[8] G. Minotti, G. Cairo, E. Monti, FASEB J. 13 (1999) $199-212$.
[9] E.L. Kostoryz, D.M. Yourtee, Mutat. Res. 490 (2001) $131-139$.

[10] K. Kiyomiya, S. Matsuo, M. Kuruebe, Cancer Chemother. Pharmacol. 47 (1) (2001) 51-56.

[11] A. Di Marco, M. Gaetani, B.M. Scarpinato, Cancer Chemother. Rep. 63 (1969) 33-45.

[12] I. Muller, A. Jenner, G. Bruchelt, D. Niethammer, B. Halliwell, Biochem. Biophys. Res. Comm. 230 (1997) 254-257.

[13] S.S. David, S.D. Williams, Chem. Rev. 98 (1998) 12211261.

[14] W.J. Pigram, W. Fuller, L.D. Hamilton, Nature (Lond.), New Biol. 235 (1972) 17-19.

[15] H. Berg, G. Horn, U. Luthardt, Bioelectrochem. Bioenerg. 8 (1981) 537-553.

[16] C.A. Frederick, L.D. Williams, G. Ughetto, G.A. Van Der Marel, J.H. Van Boom, A. Rich, A.H.J. Wang, Biochemistry 29 (1990) 2538-2549.

[17] L.A. Lipscomb, M.E. Peek, F.X. Zhou, J.A. Bertrand, D. Vanderveer, L.D. Williams, Biochemistry 33 (1994) 36493659.

[18] F. Zunino, R. Gambetta, A. Di Marco, A. Velcich, A. Zaccara, F. Quadrifoglio, V. Crescenzi, Biochim. Biophys. Acta 476 (1977) 38-46.

[19] K. Kiyomiya, S. Matsuo, M. Kuruebe, Cancer Res. 61 (6) (2001) 2467-2471.

[20] C. Cullinane, D.R. Phillips, Biochemistry 29 (1990) $5638-$ 5646.

[21] J. Wang, M. Ozsoz, X. Cai, G. Rivas, H. Shiraishi, D.H. Grant, M. Chicharro, J. Fernandes, E. Palecek, Bioelectrochem. Bioenerg. 45 (1998) 33-40.

[22] G.M. Rao, J.W. Lown, J.A. Plambeck, J. Electrochem. Soc. 125 (1978) 534-539.

[23] R.P. Baldwin, D. Packett, T.M. Woodcock, Anal. Chem. 53 (1981) 540-542.

[24] T. Konse, K. Kano, T. Kubota, J. Electroanal. Chem. 246 (1988) 385-397.

[25] A.M. Oliveira-Brett, J.A.P. Piedade, S.H.P. Serrano, Electroanalysis 12 (2000) 969-973.

[26] G. Marrazza, I. Chianella, M. Mascini, Anal. Chim. Acta 387 (1999) 297-307.

[27] A.T. Hubbard, F.C. Anson, in: A.J. Bard (Ed.), Electroanalytical Chemistry, vol. 4, Marcel Dekker, New York, 1970, pp. 129-210.

[28] C.J. Burrows, J.G. Muller, Chem. Rev. 98 (3) (1998) 1109-1151.

[29] M. Gniazdowski, C. Cera, Chem. Rev. 96 (1996) 619634.

[30] S.M. Zeman, D.R. Phillips, D.M. Crothers, Proc. Natl. Acad. Sci. USA 95 (1998) 11 561-11565.

[31] S. Steenken, Chem. Rev. 89 (1989) 503-520.

[32] H. Kasai, Z. Yamaizumi, M. Berger, J. Cadet, J. Am. Chem. Soc. 114 (1992) 9692-9694.

[33] B. Giese, Acc. Chem. Res. 33 (2000) 631-636.

[34] A. Heller, Faraday Discuss. 116 (2000) 1-13. 